\title{
ABUNDANCES IN METAL-RICH STARS
}

\author{
Detailed abundance analysis of $47 G$ and $K$ dwarf stars \\ with $[\mathrm{Me} / \mathrm{H}]>0.10 \mathrm{dex}$
}

\section{S. FELTZING \\ Royal Greenwich Observatory, Madingley Road, Cambridge CB3 OEZ, United Kingdom}

We have derived elemental abundances of $\mathrm{O}, \mathrm{Na}, \mathrm{Mg}, \mathrm{Al}, \mathrm{Si}, \mathrm{Ca}, \mathrm{Ti}$, $\mathrm{Cr}, \mathrm{Mn}, \mathrm{Fe}, \mathrm{Co}, \mathrm{Ni}$ as well as for a number of s-elements for $47 \mathrm{G}$ and $\mathrm{K}$ dwarf, with $[\mathrm{Me} / \mathrm{H}]>0.1 \mathrm{dex}$. The selection of stars was based on their kinematics as well as on their $u v b y-\beta$-photometry. One sample of stars on rather eccentric orbits traces the chemical evolution interior to the solar orbit and another, on circular orbits, the evolution around the solar orbit. A few Extreme Population I stars were included in the latter sample.

We have performed a standard (LTE) analysis to derive chemical abundances, for details see Feltzing \& Gustafsson (1997) and Feltzing (1995). Our data do, in general, fit well into the overall picture of galactic chemical evolution as it is currently understood from empirical studies. They give further constraints on theoretical models of galactic chemical evolution for the region above solar metallicity, where metallicity dependent supernova yields further complicate the picture. Our lack of success in tracing variations in relative abundances, e.g. of $[\mathrm{O} / \mathrm{Fe}],[\mathrm{Na} / \mathrm{Fe}],[\mathrm{Si} / \mathrm{Fe}],[\mathrm{Ti} / \mathrm{Fe}]$ etc to vary with the stellar velocities, i.e. with the present orbital mean distance from the galactic centre $\left(R_{\mathrm{m}}\right)$, point to a homogeneous evolution in the disk over the radius spanned. We find that the upturn in $[\mathrm{Na} / \mathrm{Fe}]$ vs $[\mathrm{Fe} / \mathrm{H}]$, as observed by Edvardsson et al. (1993), is reproduced and reinforced. In contrast to sodium we find no such upturn for aluminium. This is somewhat surprising since sodium and aluminium are thought to be produced in the same environment in the pre-supernova star.

\section{References}

Edvardsson B., Andersen J., Gustafsson B., Lambert D.L., Nissen P.E., Tomkin J. 1993a, A\&A 275, 101

Feltzing S., 1995, Thesis, "Two studies of galactic chemical evolution", Uppsala University

Feltzing S., Gustafsson, B.,1997, A\&AS, accepted, astro-ph 9710315 